\title{
A dilogarithmic integral arising in quantum field theory Djurdje Cvijović
}

\author{
Atomic Physics Laboratory, Vinča Institute of Nuclear Sciences \\ P.O. Box 522, 11001 Belgrade, Republic of Serbia \\ E-Mail: djurdje@vinca.rs
}

\begin{abstract}
Recently, an interesting dilogarithmic integral arising in quantum field theory has been closed-form evaluated in terms of the Clausen function $\mathrm{Cl}_{2}(\theta)$ by Coffey [J. Math. Phys. 49 (2008), 093508]. It represents the volume of an ideal tetrahedron in hyperbolic space and is involved in two intriguing equivalent conjectures of Borwein and Broadhurst. It is shown here, by simple and direct arguments, that this integral can be expressed by the triplet of the Clausen function values which are involved in one of the two above-mentioned conjectures.
\end{abstract}

2000 Mathematics Subject Classification. Primary 33E20, 33B30; Secondary 65B10, 11G55 . 2008 PACS number(s): 02.30.-f, 02.30.Gp, 02.30.Lt.

Key Words and Phrases. Clausen function; Dirichlet L series; Hurwitz zeta function.

\section{Introduction and statement of the results}

In a recent paper Coffey [7, Theorem 1] has evaluated the integral

$$
I_{7}=\frac{24}{7 \sqrt{7}} \int_{\pi / 3}^{\pi / 2} \ln \left|\frac{\tan (\theta)+\sqrt{7}}{\tan (\theta)-\sqrt{7}}\right| d \theta
$$

in a closed form in terms of the Clausen function $\mathrm{Cl}_{2}(\theta)$ (see Sec. 2)

$$
I_{7}=\frac{12}{7 \sqrt{7}}\left[\mathrm{Cl}_{2}\left(2 \omega_{+}\right)+2 \mathrm{Cl}_{2}\left(\theta_{+}\right)-\mathrm{Cl}_{2}\left(2 \omega_{+}+2 \theta_{+}\right)\right]
$$

where

$$
\omega_{+}=-\tan ^{-1}\left(\frac{2 \sqrt{3}+\sqrt{7}}{5}\right)=\tan ^{-1}(\sqrt{7})-\frac{2 \pi}{3} \quad \text { and } \quad \theta_{+}=\tan ^{-1}\left(\frac{\sqrt{7}}{3}\right) .
$$

This and other numerous related integrals arose out of some studies in quantum field theory, in analysis of hyperbolic manifolds whose complementary volumes result from evaluations of Feynman diagrams $[3-8,13]$. The integral $I_{7}$ is the simplest of 998 empirically determined cases where the volume of a manifold is rational multiple of values of various Dirichlet L series (see Sec. 2). $I_{7}$ was numerically computed with high accuracy (using highly parallel tanh-sinh quadrature) and represents the volume of an ideal tetrahedron in hyperbolic space.

Two intriguing equivalent conjectures concerning $I_{7}$ have arisen. First, it is conjectured by Borwein and Broadhurst [4] that 
where (see Sec. 2)

$$
I_{7} \stackrel{?}{=} \mathrm{L}_{-7}(2)
$$

$$
\begin{aligned}
\mathrm{L}_{-7}(2) & =\sum_{m=0}^{\infty}\left[\frac{1}{(7 m+1)^{2}}+\frac{1}{(7 m+2)^{2}}-\frac{1}{(7 m+3)^{2}}+\frac{1}{(7 m+4)^{2}}-\frac{1}{(7 m+5)^{2}}-\frac{1}{(7 m+6)^{2}}\right] \\
& =\frac{1}{49}\left[\zeta\left(2, \frac{1}{7}\right)+\zeta\left(2, \frac{2}{7}\right)-\zeta\left(2, \frac{3}{7}\right)+\zeta\left(2, \frac{4}{7}\right)-\zeta\left(2, \frac{5}{7}\right)-\zeta\left(2, \frac{6}{7}\right)\right] \\
& =\frac{2}{\sqrt{7}}\left[\mathrm{Cl}_{2}\left(\frac{2 \pi}{7}\right)+\mathrm{Cl}_{2}\left(\frac{4 \pi}{7}\right)-\mathrm{Cl}_{2}\left(\frac{6 \pi}{7}\right)\right] .
\end{aligned}
$$

It should be noted that equations (1.4) and (1.4') have been previously given by Coffey [7]. The ? here indicates that numerical verification (to 20,000 digit precision) has been performed but that no formal proof of this "identity" is known $[2,4]$. Second, the 1, 800 digit agreement was found (by integer relation detection algorithm) for the following unexpected relation between six values of $\mathrm{Cl}_{2}(\theta)$ (c.f. Eq. (1.4") above) [4]

$$
\mathrm{L}_{-7}(2) \stackrel{?}{=} \frac{4}{7 \sqrt{7}}\left[3 \mathrm{Cl}_{2}\left(2 \phi_{7}\right)-3 \mathrm{Cl}_{2}\left(4 \phi_{7}\right)+\mathrm{Cl}_{2}\left(6 \phi_{7}\right)\right], \quad \phi_{7}=\tan ^{-1}(\sqrt{7}) .
$$
form

In this sequel to the work of Coffey $[7,8]$ the integral $I_{7}$ is evaluated in a closed

$$
I_{7}=\frac{4}{7 \sqrt{7}}\left[3 \mathrm{Cl}_{2}\left(2 \phi_{7}\right)-3 \mathrm{Cl}_{2}\left(4 \phi_{7}\right)+\mathrm{Cl}_{2}\left(6 \phi_{7}\right)\right]
$$

by simple and direct arguments and our result, unlike (1.2a), contains one of the two triplets of the Clausen function values which are involved in the conjecture (1.5). We have been unable to deduce (1.6) from Coffey's result (1.2a) and vice versa by the usual integration techniques.

\section{Clausen function and Dirichlet $\mathrm{L}$ series}

The Clausen function (of order 2) $\mathrm{Cl}_{2}(\theta)$, sometimes also called the Clausen integral, is a real function for all $\theta \in \mathbb{R}$ and is given by ( $[14$, p. 111, Eq. (45)]

$$
\mathrm{Cl}_{2}(\theta)=\sum_{m=1}^{\infty} \frac{\sin (m \theta)}{m^{2}}=-\int_{0}^{\theta} \ln \left|2 \sin \left(\frac{t}{2}\right)\right| d t \quad(\theta \in \mathbb{R}) .
$$

The standard texts on the theory of $\mathrm{Cl}_{2}(\theta)$ (and various related functions) are Lewin's books $[11,12]$ and several new results can be found in de Doelder [9], Grosjean $[10]$ and Coffey $[7,8]$. Some elementary properties and special values of $\mathrm{Cl}_{2}(\theta)$ are:
a) $\mathrm{Cl}_{2}(-\theta)=-\mathrm{Cl}_{2}(\theta)$,
b) $\mathrm{Cl}_{2}(\theta+2 m \pi)=\mathrm{Cl}_{2}(\theta)$, for $m \in \mathbb{Z}$,
c) $\mathrm{Cl}_{2}(\pi+\theta)=-\mathrm{Cl}_{2}(\pi-\theta)$
d) $\mathrm{Cl}_{2}(m \pi)=0$, for $m \in \mathbb{Z}$. 
Define Dirichlet L series (modulo $d) \mathrm{L}_{d}(s)$ in the following way

$$
\mathrm{L}_{d}(s)=\sum_{n=1}^{\infty}\left(\frac{d}{n}\right) \frac{1}{n^{s}}
$$

where $\left(\frac{d}{n}\right)$ is the Kronecker symbol (for more details, see, for instance, [1]).

Symbol $\left(\frac{d}{n}\right)$ only assumes values $1,-1$ and 0 , it is a periodic function with a period of $|d|$ for an admissible $d$ so that $\mathrm{L}_{d}(s)$ can be expressed as the following finite sum

$$
\mathrm{L}_{d}(s)=\frac{1}{|d|^{s}} \sum_{\ell=1}^{|d|-1}\left(\frac{d}{\ell}\right) \zeta\left(s, \frac{\ell}{|d|}\right)
$$

where $\zeta(s, a)$ denotes the Hurwitz (or generalized) zeta function

$$
\zeta(s, a)=\sum_{m=0}^{\infty} \frac{1}{(m+a)^{s}} \quad(\mathfrak{R}(s)>1 ; a \notin\{0,-1,-2, \ldots\} .
$$

If $d<0$, then $\left(\frac{d}{n}\right)$ has the Fourier series expansion

$$
\left(\frac{d}{n}\right)=\frac{1}{\sqrt{|d|}} \sum_{\ell=1}^{|d|-1}\left(\frac{d}{\ell}\right) \sin \left(\frac{2 \ell n \pi}{|d|}\right) .
$$

The sequence $\left(\frac{-7}{n}\right), n \in \mathbb{N}$, is periodic in $n$ with a period of 7 , its first seven values are: $1,1,-1,1,-1,-1,0$ and its expansion is

$$
\left(\frac{-7}{n}\right)=\frac{2}{\sqrt{7}}\left[\sin \left(\frac{2 \pi}{7}\right)+\sin \left(\frac{4 \pi}{7}\right)-\sin \left(\frac{6 \pi}{7}\right)\right] .
$$

It is clear then that

$$
\begin{gathered}
\mathrm{L}_{-7}(s)=\frac{1}{7^{s}}\left[\zeta\left(s, \frac{1}{7}\right)+\zeta\left(s, \frac{2}{7}\right)-\zeta\left(s, \frac{3}{7}\right)+\zeta\left(s, \frac{4}{7}\right)-\zeta\left(s, \frac{5}{7}\right)-\zeta\left(s, \frac{6}{7}\right)\right] \\
=\sum_{m=0}^{\infty}\left[\frac{1}{(7 m+1)^{s}}+\frac{1}{(7 m+2)^{s}}-\frac{1}{(7 m+3)^{s}}+\frac{1}{(7 m+4)^{s}}-\frac{1}{(7 m+5)^{s}}-\frac{1}{(7 m+6)^{s}}\right],
\end{gathered}
$$

so that for $s=2$ we have the values of $\mathrm{L}_{-7}(2)$ given by (1.4) and (1.4'). In addition, the value of $\mathrm{L}_{-7}(2)$ in terms of the Clausen function (1.4") follows from (2.1), (2.2) and (2.4).

\section{Proof of equation (1.6)}

In order to evaluate the integral given by equation (1.6) we shall need the following two results. The Lemma 2 is familiar multiplication formula for the Clausen function $\mathrm{Cl}_{2}(\theta)$ (see, for instance, [12, p. 105, Eq. (4.24)]) and we include it and its proof for the sake of self-containedness. In addition, both integrals, (3.1) and (3.2), given by Lemma 1 are essentially the same as the integral in (3.5) below, which, in turn, can be found in well-known books by Lewin $[11,12]$ (see, for instance, [12, p. 308, Eq. $(37)])$. 
Lemma 1. In terms of the Clausen function $\mathrm{Cl}_{2}(\theta)$ defined as in (2.1), we have:

a) $\quad \int_{\phi}^{x} \ln \left(\frac{\tan (\theta)+\tan (\phi)}{\tan (\theta)-\tan (\phi)}\right) d \theta$

$$
=-\frac{1}{2} \mathrm{Cl}_{2}(2 x+2 \phi)+\frac{1}{2} \mathrm{Cl}_{2}(2 x-2 \phi)+\frac{1}{2} \mathrm{Cl}_{2}(4 \phi) ;
$$

b) $\quad \int_{x}^{\phi} \ln \left(\frac{\tan (\phi)+\tan (\theta)}{\tan (\phi)-\tan (\theta)}\right) d \theta$

$$
=\frac{1}{2} \mathrm{Cl}_{2}(2 x+2 \phi)-\frac{1}{2} \mathrm{Cl}_{2}(2 x-2 \phi)-\frac{1}{2} \mathrm{Cl}_{2}(4 \phi) .
$$

Lemma 2 (Multiplication formula). For $m \in \mathbb{N}$, we have

$$
\mathrm{Cl}_{2}(m \theta)=m \sum_{\ell=0}^{m-1} \mathrm{Cl}_{2}\left(\theta+\ell \frac{2 \pi}{m}\right) .
$$

In particular

$$
\frac{1}{2} \mathrm{Cl}_{2}(2 \theta)=\mathrm{Cl}_{2}(\theta)+\mathrm{Cl}_{2}(\pi+\theta)=\mathrm{Cl}_{2}(\theta)-\mathrm{Cl}_{2}(\pi-\theta)
$$

and

$$
\frac{1}{3} \mathrm{Cl}_{2}(3 \theta)=\mathrm{Cl}_{2}(\theta)+\mathrm{Cl}_{2}\left(\theta+\frac{2 \pi}{3}\right)+\mathrm{Cl}_{2}\left(\theta-\frac{2 \pi}{3}\right) .
$$

To prove Lemma 1, first observe that $\ln [\tan (\theta)+\tan (\phi)]$ can be rewritten as follows

$$
\begin{aligned}
\ln [\tan (\theta)+\tan (\phi)]=\ln \left[\frac{\sin (\theta+\phi)}{\cos (\theta) \cos (\phi)}\right] \\
=\ln [\sin (\theta+\phi)]-\ln \left[\sin \left(\frac{\pi}{2}-\theta\right)\right]-\ln [\cos (\phi)] \\
=\ln \left[2 \sin \left(\frac{2 \theta+2 \phi}{2}\right)\right]-\ln \left[2 \sin \left(\frac{\pi-2 \theta}{2}\right)\right]-\ln [\cos (\phi)]
\end{aligned}
$$

and consequently

$$
\begin{aligned}
\int \ln [\tan (\theta)+\tan (\phi)] d \theta & =-\ln [\cos (\phi)] \theta+\frac{1}{2} \int \ln \left[2 \sin \left(\frac{u}{2}\right)\right] d u \\
& +\frac{1}{2} \int \ln \left[2 \sin \left(\frac{v}{2}\right)\right] d v+C
\end{aligned}
$$

where

$$
u=2 \theta+2 \phi \quad \text { and } \quad v=\pi-2 \theta,
$$

so that the above integral, in view of the definition of $\mathrm{Cl}_{2}(\theta)$ in (2.1), at once yields 


$$
\begin{aligned}
\int \ln [\tan (\theta)+\tan (\phi)] d \theta= & -\ln [\cos (\phi)] \theta \\
& -\frac{1}{2} \mathrm{Cl}_{2}(2 \theta+2 \phi)-\frac{1}{2} \mathrm{Cl}_{2}(\pi-2 \theta)+C .
\end{aligned}
$$

Finally, upon noting that $\mathrm{Cl}_{2}(0)=0$ (see the definition of $\mathrm{Cl}_{2}(\theta)$ in $(2.1)$ ), the required formulae (3.1) and (3.2) are readily available from (3.5).

It should be noted that Coffey $[8$, Sec. 6$]$ has evaluated around dozen new integrals that are expressible in terms of the function $\mathrm{Cl}_{2}(x)$, examples of which are

$$
\begin{aligned}
& \kappa \int_{0}^{x} \ln [\sin (\kappa \theta)+\sin (\alpha)] d \theta \\
& =\mathrm{Cl}_{2}(\alpha)-\mathrm{Cl}_{2}(\kappa x+\alpha)+\mathrm{Cl}_{2}(\alpha-\kappa x+\pi)-\mathrm{Cl}_{2}(\alpha+\pi)-x \kappa \ln 2
\end{aligned}
$$

for $\kappa>0$ and $|x| \leq|\alpha|$, and

$$
-\kappa \int_{0}^{x} \ln |\cos (\alpha)-\cos (\kappa \theta)| d \theta=\mathrm{Cl}_{2}(\kappa x-\alpha)+\mathrm{Cl}_{2}(\kappa x+\alpha)+x \kappa \ln 2,
$$

for $\alpha \in \mathbb{R}$, but (3.5) as well as (3.1) and (3.2) are not included in his analysis.

To prove Lemma 2 we apply the well-known trigonometric formula

$$
\sum_{\ell=0}^{m-1} \sin \left[n\left(\theta+\ell \frac{2 \pi}{m}\right)\right]=\left\{\begin{array}{ll}
0, & \text { if } m \nmid n \\
m \sin (n \theta) & \text { if } m \mid n
\end{array} .\right.
$$

Hence

$$
\begin{aligned}
\sum_{\ell=0}^{m-1} \mathrm{Cl}_{2}\left(\theta+\ell \frac{2 \pi}{m}\right) & =\sum_{n=1}^{\infty} \sum_{\ell=0}^{m-1} \frac{1}{n^{2}} \sin \left[n\left(\theta+\ell \frac{2 \pi}{m}\right)\right] \\
& =\sum_{\substack{n=1 \\
m \mid n}}^{\infty} \frac{m}{n^{2}} \sin (n \theta)=\sum_{\ell=1}^{\infty} \frac{m}{(m \ell)^{2}} \sin (m \ell \theta)=\frac{1}{m} \mathrm{Cl}_{2}(m \theta)
\end{aligned}
$$

We conclude this section with the proof of (1.6). Indeed, we may make use of Lemma 1 since the integral $I_{7}$ could be decomposed as

$$
\frac{7 \sqrt{7}}{24} I_{7}=\int_{\pi / 3}^{\phi_{7}} \ln \left(\frac{\tan \left(\phi_{7}\right)+\tan (\theta)}{\tan \left(\phi_{7}\right)-\tan (\theta)}\right) d \theta+\int_{\phi_{7}}^{\pi / 2} \ln \left(\frac{\tan (\theta)+\tan \left(\phi_{7}\right)}{\tan (\theta)-\tan \left(\phi_{7}\right)}\right) d \theta
$$

$\phi_{7}=\tan ^{-1}(\sqrt{7}) \approx 1.209429202888189$ being the only singularity inside the interval $\{\pi / 3, \pi / 2\}$, and in this way we arrive at

$$
\frac{7 \sqrt{7}}{24} I_{7}=\frac{1}{2}\left[\mathrm{Cl}_{2}\left(2 \phi_{7}+\frac{2 \pi}{3}\right)+\mathrm{Cl}_{2}\left(2 \phi_{7}-\frac{2 \pi}{3}\right)\right]-\mathrm{Cl}_{2}\left(\pi+2 \phi_{7}\right) .
$$


On the other hand, by means of the triplication and duplication formulae, (3.3) and (3.4), we have

$$
\mathrm{Cl}_{2}\left(2 \phi_{7}+\frac{2 \pi}{3}\right)+\mathrm{Cl}_{2}\left(2 \phi_{7}-\frac{2 \pi}{3}\right)=\frac{1}{3} \mathrm{Cl}_{2}\left(6 \phi_{7}\right)-\mathrm{Cl}_{2}\left(2 \phi_{7}\right)
$$

and

$$
\mathrm{Cl}_{2}\left(\pi+2 \phi_{7}\right)=\frac{1}{2} \mathrm{Cl}_{2}\left(4 \phi_{7}\right)-\mathrm{Cl}_{2}\left(2 \phi_{7}\right)
$$

thus from (3.7) it follows

$$
\frac{7 \sqrt{7}}{24} I_{7}=\frac{1}{6}\left[3 \mathrm{Cl}_{2}\left(2 \phi_{7}\right)-3 \mathrm{Cl}_{2}\left(4 \phi_{7}\right)+\mathrm{Cl}_{2}\left(6 \phi_{7}\right)\right]
$$

which is the sought result given by (1.6).

\section{References}

[1] R.G. Ayoub, An Introduction to the Analytic Theory of Numbers, American Mathematical Society, Providence 1963.

[2] D.H. Bailey and J.M. Borwein, Computer-Assisted Discovery and Proof, in T. Amdeberhan and V. Moll (eds.), Tapas in Experimental Mathematics, Contemporary Mathematics, vol 457, American Mathematical Society, 2008, pp. 21-52.

[3] J.M. Borwein, I.J. Zucker, and J. Boersma, The evaluation of character Euler double sums, Ramanujan J. 15 (2008), 377-405.

[4] J.M. Borwein and D.J. Broadhurst, Determinations of rational Dedekind-zeta invariants of hyperbolic manifolds and Feynman knots and links, e-print arXiv:hep-th/9811173.

[5] D.J. Broadhurst, Solving differential equations for three-loop diagrams: relation to hyperbolic geometry and knot theory, e-print http://xxx.lanl.gov/abs/hep-th/9806174

[6] D.J. Broadhurst, Massive 3-loop Feynman diagrams reducible to $\mathrm{SC}^{*}$ primitives of algebras of the sixth root of unity, Eur. Phys. J. C 8 (1999), 311-333.

[7] M.W. Coffey, Evaluation of a ln tan integral arising in quantum field theory, J. Math. Phys. 49 (2008), 093508.

[8] M.W. Coffey, On a three-dimensional symmetric Ising tetrahedron and contributions to the theory of the dilogarithm and Clausen functions, J. Math. Phys. 49 (2008), 043510.

[9] P.J. de Doelder, On the Clausen integral $\mathrm{Cl}_{2}(\theta)$ and a related integral, J. Comput. Appl. Math. 11 (1984), 325-330.

[10] C.C. Grosjean, Formulae concerning the computation of the Clausen integral $\mathrm{Cl}_{2}(\theta)$, J. Comput. Appl. Math. 11 (1984), 331-342.

[11] L. Lewin, Dilogarithms and Associated Functions, Macdonald, London, 1958.

[12] L. Lewin, Polylogarithms and Associated Functions, North-Holland, New York, 1981.

[13] F.A. Lunev, Evaluation of two-loop self-energy diagram with three propagators, Phys. Rev. D 50 (1994), 7735-7737.

[14] H.M. Srivastava and J. Choi, Series associated with the zeta and related functions, Kluwer Academic Publishers, Dordrecht, 2001. 